\title{
Study on Effects of Polymer for SP Binary Flooding on Emulsion Flowing Rules in Porous Medium
}

\author{
Jianlong Xiu ${ }^{1,2, a}$, Weiyao $\mathrm{Zhu}^{1, \mathrm{~b}}$, Ying Guo ${ }^{2, \mathrm{c}}$, Li Yu $\mathrm{Lu}^{2, \mathrm{~d}}$ \\ ${ }^{1}$ Civil and Environmental Engineering School ,University of Science and Technology Beijing, Beijing \\ 100083 China \\ ${ }^{2}$ Langfang Branch of PetroChina Research Institute of Petroleum Exploration \& Development, \\ Langfang,065007 China;

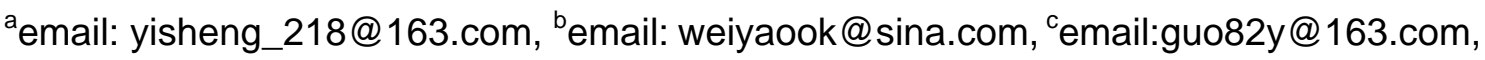 \\ demail: yuli69@petrochina.com.cn
}

Keywords: Polymer-Surfactant Binary Flooding; Polymer; Shear Force; Porous Medium; Velocity

\begin{abstract}
In surfactant-polymer binary flooding, to study the effects of polymer in emulsion seepage flow rules in porous medium, artificial sandstone cores were established to carry out the experimental analysis. Biology microscope and sample analytic control methods were employed for all samples. Results displayed that polymer can increase the strength of the interfacial film, and water phase viscosity would increase with the existence of the addition polymer, which reduces the floating speed of emulsified droplets and boosts the emulsion stability. The bigger polymer relative molecular weight is, the easier molecular chain intertwines. With the shear force in porous media, polymer has been cut into small molecular chain segments and the diffusivity of emulsion droplets increases. The multilayer three-dimensional network structure in the polymer solution contributes to form $\mathrm{O} / \mathrm{W}$ emulsion. Under the interfacial tension of 10-3 grade, porous media has a strong shearing effect on fluid. As the velocity gets larger, the initially status dominated by two phases fluid flow is transformed into emulsion flow mode gradually.
\end{abstract}

\section{Introduction}

In oilfield application to improve crude oil recovery for surfactant-polymer (SP) flooding, the emulsion for oil and water two phases in porous medium has appealed much more attention in recent years. The practical emulsion phenomenon conduces to the large amounts of oil produced in oilfield. Emulsion is an important mechanism [1][2][3], and relative studies have displayed that the chemical type, concentration, polymer, flow velocity, properties of crude oil are the key factors affecting emulsion [4][5][6]. On the one hand, porous media would destroy the structure of droplet, resulting in demulsification; on the other hand, it can cut large droplets into small ones and emulsion droplet distribution are more uniform by promoting emulsification [7].

The structure of porous pore media also influences the distribution range of emulsion droplets. In actual process of seepage flow, the comprehensive effect for velocity and porous media mainly focuses on the chemical stability of the emulsion system itself and the shear force which controls the emulsification. For a certain emulsion system, its chemical stability is fixed too. The seepage velocity is controlled by shear force of emulsion droplet and polymer. The pore throat collocation and droplet size have become the important parameters in emulsion's seepage for porous media [8][9][10]. With high viscosity, polymer is a main factor in the formation of emulsion, but no specific study is conducted in the O/W type emulsion, Therefore, in view of the current situation, mechanisms of polymer were conducted for emulsion formation in SP binary flooding.

\section{Experimental Process and Methods}

For the great majority oilfields in China, the water cut is high enough in tertiary oil recovery, and it is easy to form the $\mathrm{O} / \mathrm{W}$ type emulsion in most cases, so this research mainly focus on the O/W emulsion. In this study, artificial sandstone cores were used to carry out this experimental research. 
The length of these cores is $30.0 \mathrm{~cm}$, and the inner diameter is $2.0 \mathrm{~cm}$. The medium permeability of these cores are mainly distributed in $200-500 \times 10^{-3} \mu \mathrm{m}^{2}$. Under the condition of unsaturated oil, simulated oil and binary mixture were injected into the cores with a certain ratio of oil/water. To guarantee the ratio of oil-water mixture is always constant, premix is conducted in this research. With different conditions of injection parameters, the two phases of oil and water would gradually displace the stratum water in the main flowing pore throat of cores, and form some fixed pore passages. When this flow reaches one steady state, samples were collected and then these relative parameters were tested.

To study the effect of velocity for emulsion in porous media seepage law, the mixture was injected into the cores by different rates of $0.04 \mathrm{~m} / \mathrm{d}, 0.19 \mathrm{~m} / \mathrm{d}, 0.38 \mathrm{~m} / \mathrm{d}, 1.00 \mathrm{~m} / \mathrm{d}, 3.19 \mathrm{~m} / \mathrm{d}, 6.39$ $\mathrm{m} / \mathrm{d}, 12.78 \mathrm{~m} / \mathrm{d}, 25.56 \mathrm{~m} / \mathrm{d}$ and $51.12 \mathrm{~m} / \mathrm{d}$. The viscosity of mixture (oil and water phases) is 85.2 $\mathrm{mPa}$.s after preliminary premix. The formulation of binary system is $1 \% \mathrm{DWS}-3+0.12 \% \mathrm{HPAM}$, and its interfacial tension is $1.89 \times 10^{-3} \mathrm{mN} / \mathrm{m}$. The experimental procedure was shown as following.

I. Artificial sandstone cores should be made and selected with their permeability, and the parameters such as length, diameter and quality should be measured;

II. In vacuum state by pumping out the air, these cores are saturated with stratum water for 24 hours. The next steps are quality measurement and calculating their porosity and permeability;

III. Oil-water mixture are injected into the cores with the ratio of oil-water 3:7 after preliminary mixing. The initial injection rate is $0.1 \mathrm{~mL} / \mathrm{min}$, and the injection rate should increase gradually in subsequent experiments;

IV. Under different flow speed, samples are collected in the state of pressure stability. It should be confirmed that whether there is a phenomenon of emulsion. Then the pressure, flow rate, sample parameters such as the volume of each phase are recorded too;

V. Emulsion samples are analyzed. The end of the experiment.

\section{Results and Analysis}

Polymer used in this experiment is KY-1, and its concentration is $0.12 \%$. On the condition of $40{ }^{\circ} \mathrm{C}$ temperature, the viscosity of polymer was monitored and tested, as shown in figure 1 . Rules of viscosity under different concentrations for polymer are offered in figure 2. Polymer solution viscosity has reduced by $13.67 \%$ after 50 days. The value change about viscosity rate is acceptable, so the polymer aging properties conform to the requirements of the application.

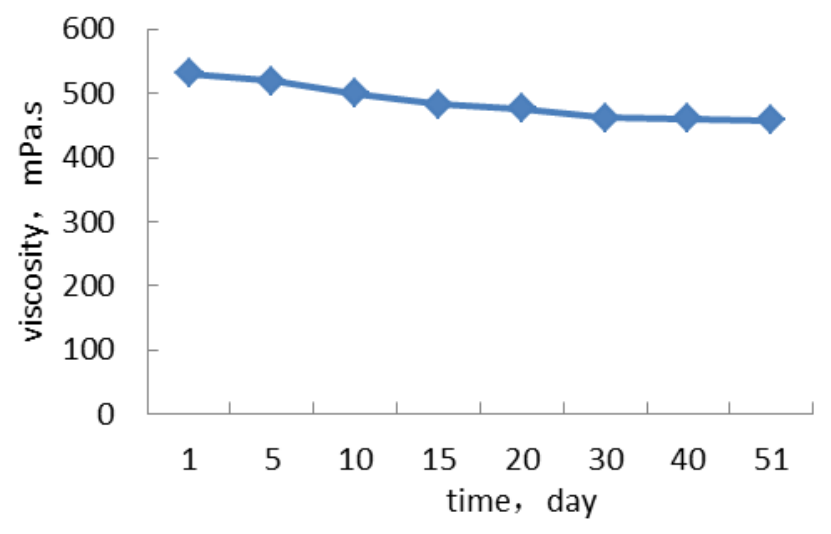

Fig.1. Rules of viscosity with time for polymer 


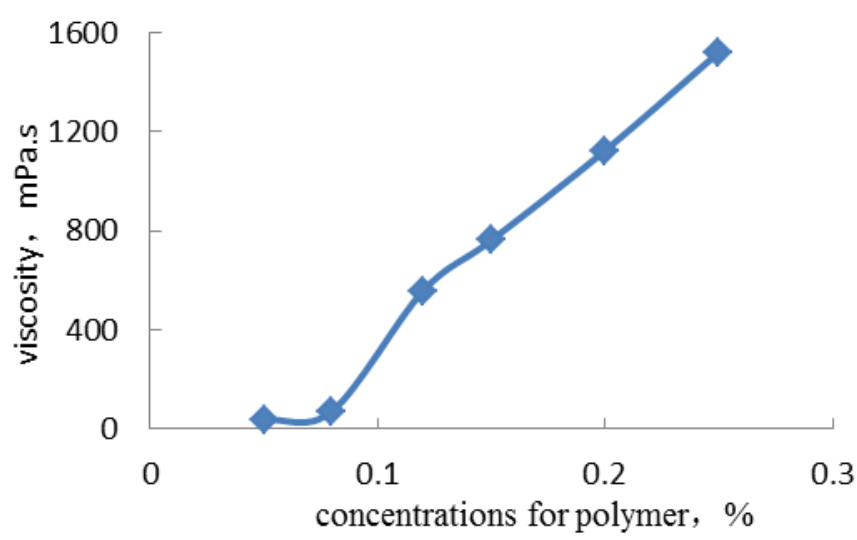

Fig.2. Rules of viscosity under different concentrations for polymer

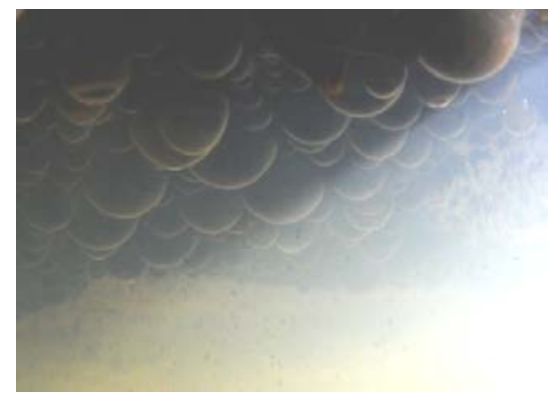

Fig.3. Droplet shape image in interface between oil and water two phases

In the process of emulsion preparation with polymer-surfactant binary flooding, it was found that in preliminary mix there was a layer of interface with a large number of semicircle droplets between oil and water phases without shear force, which was shown in figure 3 . This phenomenon may be due to the macromolecular polymer solution which increased the intensity of interfacial film. Macromolecular polymer has the ability of stabilize droplet.

Because polymer can increase the strength of the interfacial film, the emulsion is not easy to gather and to be demulsified. The bigger polymer relative molecular weight is, the easier molecular chain intertwines. Under bigger shear force, the size of droplets could decrease greatly, and the macromolecule polymer chain can be cut into small ones. At the same time, for the water soluble polymer, it has good solubility in the aqueous solution, therefore, the dissolved polymer is easy to become a continuous phase of water phase, and the oil phase becomes dispersed phase in the formation of $\mathrm{O} / \mathrm{W}$ emulsion.

According to the results from related research [11][12], in porous medium under the action of shear force, two phase viscosity ratio (dispersed phase and continuous phase) is closely related to the stability of the emulsion. For O/W emulsion, water phase viscosity would increase because of the addition of polymer, helping to form $\mathrm{O} / \mathrm{W}$ emulsion. After being sheared, the micro droplet distribution were shown in figure 4.

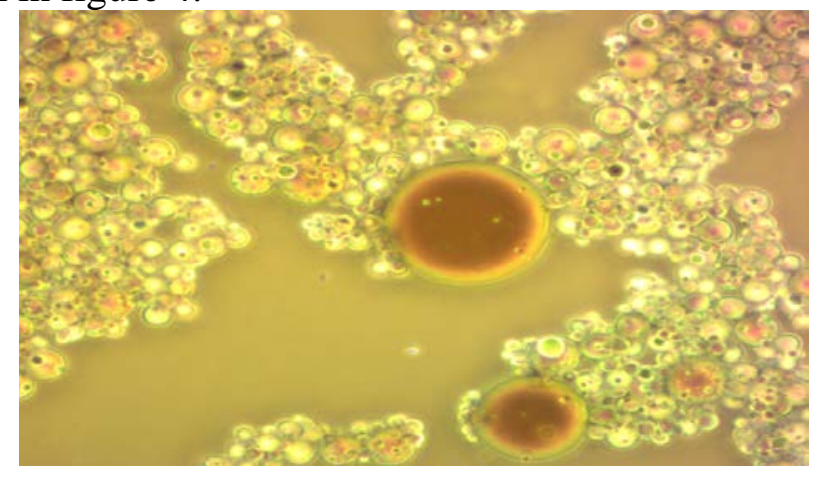

Fig.4. Micro distribution $\mathrm{n}$ of droplet and polymer molecules after shearing (The smaller faint yellow droplets are molecular chain segments, and the bigger brown circles are oil droplets)

For W/O emulsion, polymer would increase the viscosity and stability of water phase. Polymer is 
difficult to be cut into small segments and is very hard to be broken up, so polymer would hinder the formation of emulsion. Stokes principle analysis has showed that the particle size of oil droplets, the density difference between oil and water phase, water phase viscosity are the main factors influencing the rate of oil droplets floating rate. If the oil droplet size, the density difference between oil and water phase are greater or the water phase viscosity is smaller, the oil droplets rises faster. Polymer can increase the water phase viscosity and reduce the floating speed of emulsified droplets, boosting the emulsion stability. These above principles can explain the phenomenon of nice emulsification stability with produced liquid samples from oilfield's polymer flooding and SP flooding.

In the microscopic morphological analysis for polymer molecular, surfactant has a main impact on hydrodynamic diameter of polymer molecule. With polymer-surfactant binary flooding solution, large molecules polymer would present the state of stretching. The macromolecular easily intertwine around each other. There is a multilayer three-dimensional network structure in the polymer solution. This structure contains a different size of holes, and the hole density is very big. The supportive network structure has led to the adsorption and package of many water molecules to produce deformation resistance. Polymer solution is in a good ability of increasing viscosity. With the join of sulfonic acid salts in polymer solution, anionic ionization of hydrophobic groups and $\mathrm{Na}^{+}$ could compress the polymer molecular thread. The space skeleton of polymer molecules is sparse. Due to there is a certain repulsion between the large molecules polymer chain and hydrophobic group, the molecular chain presents the state of relaxing [13], which increases the molecular thermal motion and the molecular diameter of fluid mechanics.

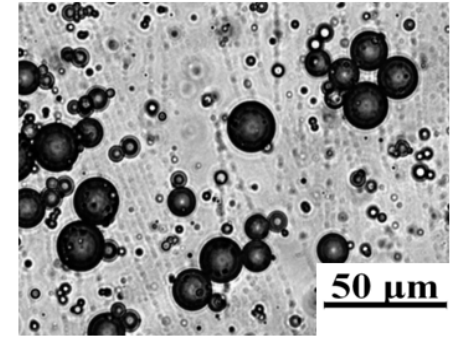

(a)

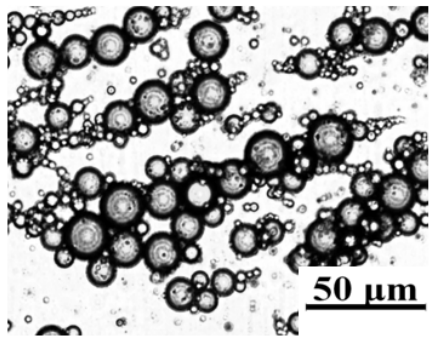

(b)

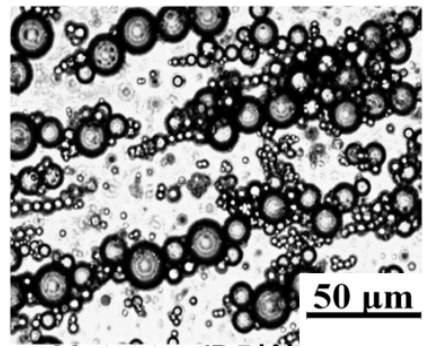

(c)

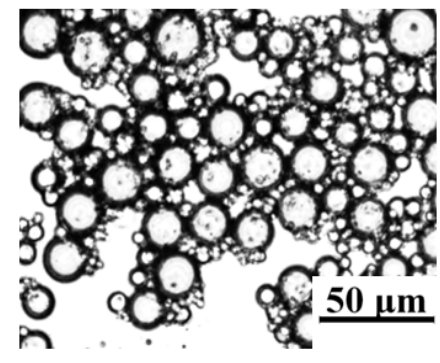

(d)

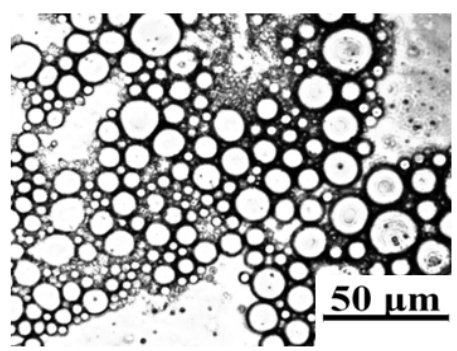

(e)

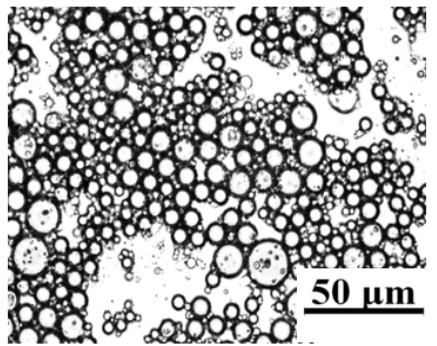

(f)

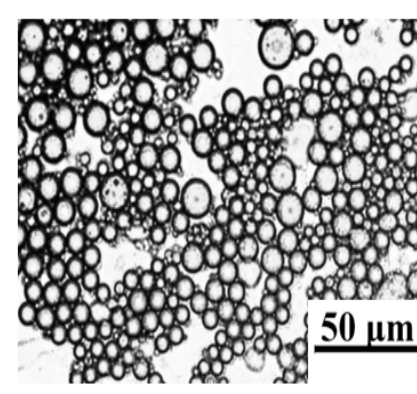

(g)

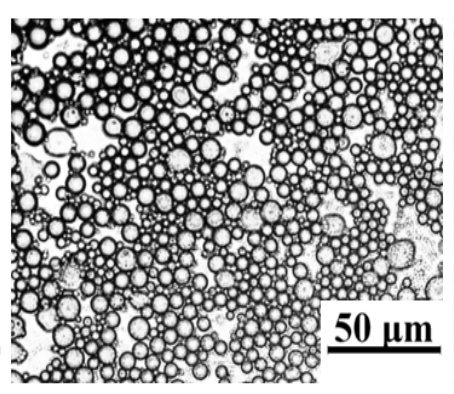

(h)

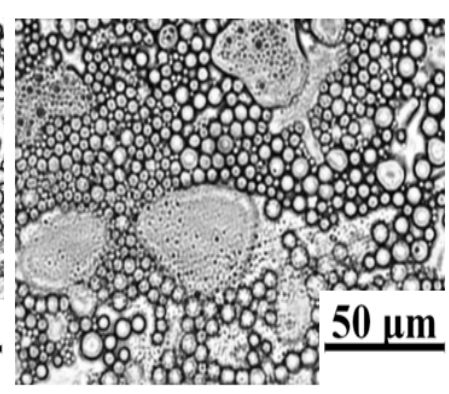

(i)

Fig.5. Microscopic images for emulsion samples $(a, b 、 c, d, e, f 、 g 、 h 、 i$ are represent the velocity of $0.04 \mathrm{~m} / \mathrm{d} 、 0.19 \mathrm{~m} / \mathrm{d} 、 0.38 \mathrm{~m} / \mathrm{d} 、 1.00 \mathrm{~m} / \mathrm{d} 、 3.19 \mathrm{~m} / \mathrm{d} 、 6.39 \mathrm{~m} / \mathrm{d} 、 12.78 \mathrm{~m} / \mathrm{d} 、 25.56 \mathrm{~m} / \mathrm{d} 、 51.12$ $\mathrm{m} / \mathrm{d}$ respectively) 
With the increase of flow velocity, experimental results have showed that the proportion of medium and small droplets increases rapidly while the large droplets proportion adds slowly. Microscopic images are shown in figure 5 for different emulsion samples. Under the low velocity $\mathrm{V}$ $\leqslant 0.38 \mathrm{~m} / \mathrm{d}$, because the molecular chain of polymer is longer without high shear strength, the stability of droplet is strong. The formation of emulsion drops are less with the clusters of distribution, and its diffusivity is poor too. In the middle velocity of $0.38 \mathrm{~m} / \mathrm{d}<\mathrm{V}<12.78 \mathrm{~m} / \mathrm{d}$, with more polymer shear strengthen and smaller molecular chain length, droplet dispersion increases gradually. With high velocity $\mathrm{V} \geqslant 12.78 \mathrm{~m} / \mathrm{d}$, polymer has been cut into small molecular chain segments and the diffusivity of emulsion droplets increases. The quantity of liquid droplets adds rapidly, also the proportion of small droplets increases rapidly while the large droplets proportion adds slowly. When $\mathrm{V}$ is about $51.12 \mathrm{~m} / \mathrm{d}$, the proportional of small droplets is about $97 \%$. As the velocity gets larger, the oil and water two phases fluid flow dominated initially is transformed into emulsion flow mode. The density and diffusivity of emulsion droplets increases gradually.

\section{Conclusion}

In SP binary flooding with the addition of polymer, the strength of interfacial film and water phase viscosity would increase, and in this case the floating speed of emulsified droplets reduces, the emulsion stability boosts for the above serial variation. The bigger polymer relative molecular weight gets, the easier molecular chain intertwines. Polymer has been cut into smaller molecular chain segments by the shear force in porous media, and the diffusivity of emulsion droplets increases. The multilayer three-dimensional network structure in the polymer solution contributes to the formation of $\mathrm{O} / \mathrm{W}$ emulsion. Porous media has a strong shearing action on fluid. As the velocity gets larger, the oil and water two phases fluid flow dominated initially is transformed into emulsion flow mode gradually.

\section{Acknowledgement}

The research work was supported by National Science and Technology Major Project of China under Grant No. 2011ZX05031. Thanks to the employee and students from Institute of Porous flow \& Fluid Mechanics.

\section{References}

[1] Kang Wan-li. Research on chemical mechanism for ASP flooding in Daqing oilfield. Beijing: Petroleum Industry Press, 96-120.(2001)

[2] Liu Yi, Zhang Zi-han, Liao Guang-zhi. The effect of crude oil emulsion on enhanced oil recovery in alkaline surfactant polymer flooding. Detergent \&cosmetics. 23, 124-127.(2000)

[3] Wang Jian, Leng Dong-mei, LIU Wei. The latest technology and related evaluation methods for crude oil emulsion preparation. Oil-gas field Surface Engineering. 31, 38-39. (2012)

[4] Kang Wan-li, Li Jin-huan, Zhao Xue-qian. Effect of interfacial tension and droplet size on the stability of emulsion. Oil-gas field Surface Engineering. 24, 11-12.(2005)

[5] Kang Wan-li, Yue Xiang-an, Hu Jing-bang. A study on stability of crude emulsion containing polymer. Oil-gas field Surface Engineering. 14, 28-30.(1995)

[6] Yu Da-sen, Huang Yan-zhang, Chen Quan. Structural changes of crude oil-in-water emulsions in viscosimetric flow. Oilfield chemistry. 9, 348-351.(1992)

[7] Kang Wan-li, Li Jin-huan, Liu Gui-fan. Preparation and stability of simulated crude-oil emulsion. Journal of northeast normal university(Natural Science). 37, 76-79. (2005)

[8] Meng Xiang-peng, Pan Yuan-yuan, Wang Shu-he. Research on low oil content of crude oil emulsion preparation technology. Chemical engineering \&equipment. 11, 7-9.( 2011) 
[9] David Cuthiell, Kirk Green. The in situ formation of heavy oil emulsion. 1995 SPE30319, 675-685.

[10] Zhu Bin, Hong Ying. Emulsion microstructure from digital image analysis technique. Petroleum exploration and development. 31,52-54.(2004)

[11] Briscoe B J, Lawrence C J, Mietus W G P. A review of immiscible fluid mixing. Advances of Colloid and Interface Science. 81,1-17.(1999)

[12] Wu Di, Meng Xiang-chun, Zhao Feng-ling.: Emulsification and stabilization of ASP flooding produced liquid. SPE 65390.(2001)

[13] Lu Xiang-guo, Jiang Wei-dong, Wang Xiao-yan. Study on effects of Cr3+,alkali and surfactant on polymer molecular configuration and seepage flow characteristics . Acta petrolei sinica, 30, 749-754. (2009) 